\title{
Assessing cross-presentation in vivo
}

The capacity of dendritic cells (DCs) to cross-present exogenous antigens on MHC class I molecules is well established, although the importance of cross-presentation for different types of immune response in vivo is not clear. In a recent report published in Science, Murphy and colleagues show that deficiency in the transcription factor BATF3 (basic leucine zipper transcription factor, ATF-like 3) leads to impaired immune responses in vivo owing to the absence of a DC subset that is particularly important for cross-presentation.

The authors used global gene expression analysis to search for genes that might be involved in the development of different DC subsets and found that BATF3 was highly expressed by conventional DCs (cDCs). Deletion of Batf3 in mice caused a selective loss of the $\mathrm{CD} 8 \alpha^{+}$subset of $\mathrm{cDCs}$ in the spleen without affecting the development of other haematopoietic cell types.

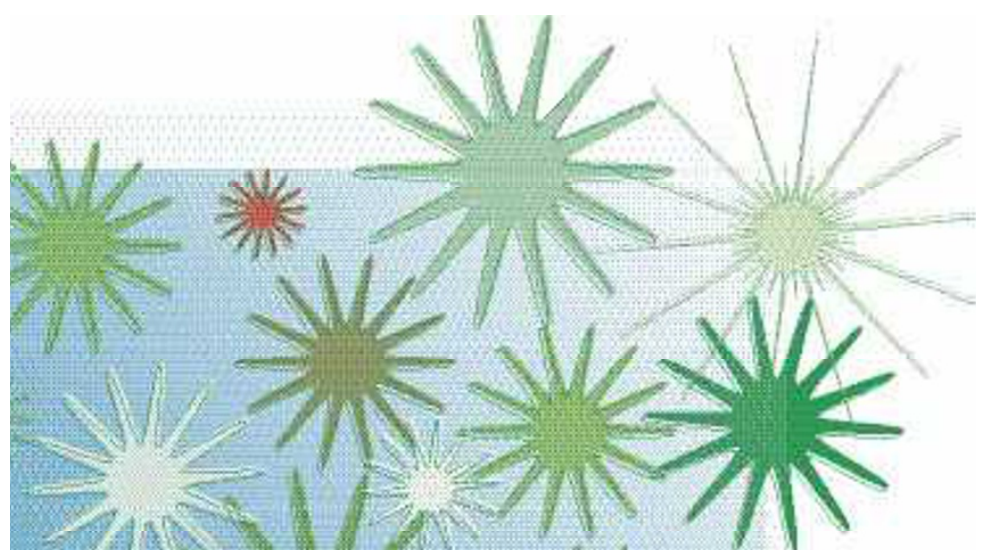

CD8 $\alpha^{+}$cDCs are characterized by their efficient ability to cross-present antigen and their responsiveness to Toll-like receptor 3 (TLR3) ligands. Indeed, when the function of $\mathrm{cDCs}$ from $B a t f 3^{-/-}$and wild-type mice was compared, those from the mutant mice showed marked impairments in TLR3-mediated cytokine production and antigen cross-presentation in vitro.

Having established that the transcription factor BATF3 is required for $\mathrm{CD} 8 \alpha^{+} \mathrm{CDC}$ development in mice, the authors next investigated how a deficiency in this DC subset influenced immune responses in which cross-presentation is important in vivo. First, using a model of West Nile virus (WNV) infection, the authors found that Batf $3^{-/-}$mice had a markedly impaired $\mathrm{CD} 8^{+} \mathrm{T}$-cell response, but had normal B-cell and $\mathrm{CD}^{+} \mathrm{T}$-cell responses to WNV. Second, the authors investigated the importance of CD $8 \alpha^{+} \mathrm{cDCs}$ in tumour immunity. Wild-type mice could reject syngeneic fibrosarcomas, whereas these tumours grew rapidly in $\mathrm{Batf3}^{-/-}$mice. The failure to reject the tumours was associated with reduced infiltration of $\mathrm{CD}^{+}$but not $\mathrm{CD} 4^{+} \mathrm{T}$ cells into the tumours and an impaired development of tumour-specific CD8 ${ }^{+} \mathrm{T}$ cells.

To rule out the possibility that the impaired antiviral and antitumour responses in $\mathrm{Batf3}^{-/-}$mice were due to intrinsic T-cell defects, the authors carried out several assays of T-cell differentiation and function. Defects in Batf3 $3^{--}$T cells could not be detected, which supported the authors' conclusion that impaired antiviral and antitumour responses in $\mathrm{Batf3}^{-/-}$mice were due to the lack of CD $8 \alpha^{+}$cDCs. However, a closer examination of antitumour responses in $\mathrm{Batf}^{-/-}$mice indicated that immune-cell populations other than $\mathrm{CD} 8 \alpha^{+} \mathrm{cDC}$ were sufficient to promote an antitumour response in mice that carried a low tumour burden. Therefore, although cross-presentation that is mediated by $\mathrm{CD} 8 \alpha^{+} \mathrm{cDCs}$ is important for optimal priming of $\mathrm{CD} 8^{+} \mathrm{T}$-cell responses, other subsets of antigenpresenting cells can also carry out this function in vivo.

This work shows that BATF3 is required for the development of CD $8 \alpha^{+}$cDCs, a subset with crosspresenting ability that is required for optimal immune responses to viral infections and tumours in mice.

Sarah Allan

ORIGINAL RESEARCH PAPER Hildner, K. et al. Batf3 deficiency reveals a critical role for $\mathrm{CD} 8 \alpha^{+}$ dendritic cells in cytotoxic T cell immunity. Science 322, 1097-1100 (2008) 